\title{
EFFECT OF SOME FERTILIZATION TREATMENTS ON GROWTH OF TREES, PRODUCTIVITY AND QUALITY OF GUAVA (Psidium guajava I.) FRUITS CV. "ETMANI". I. UNDER THE CONVENTIONAL FLOOD IRRIGATION SYSTEM. \\ Mekhiel, E.G. ; I.M.S. Osman and A.A. El-Taweel \\ Olive and Fruits of Semiarid Zone Dept., Hort. Res. Inst., ARC, Giza, Egypt.
}

\begin{abstract}
A set of field experiments was conducted in a private orchard at Qalyob district, Qalubia governorate, Egypt during 2012, 2013 and 2014 seasons of 6-years-old uniform trees of guava (Psidium guajava L.) cv. Etmani grown on clay loam soil at $5 \mathrm{x}$ $5 \mathrm{~m}$ apart and irrigated with the ordinary flood system to study the response of trees to organic compost at either full, $3 / 4$ or $1 / 2$ the recommended dose $(40,30$ and $20 \mathrm{~kg} / \mathrm{tree}$, respectively) + feldspar at either full, $3 / 4,1 / 2$ or $1 / 4$ the recommended dose $(1200,900$, 600 and $300 \mathrm{~g} /$ tree, respectively) + rock phosphate at either full, $3 / 4,1 / 2$ or $1 / 4$ the recommended dose $(1300,975,650$ and $325 \mathrm{~g} /$ tree, respectively) + mixture of bisofertilizers (Nitropeine + phosphoreine + potasseine at 120, $25 \mathrm{~g}$. and $134 \mathrm{~cm}$./tree, respectively) in 12 combined treatments, besides the control.

The obtained results indicated that all fertilization treatments used in this study caused a marked improvement in the means of shoot length, number of leaves $/ \mathrm{m}$, leaf area and number of flower buds $/ \mathrm{m}$ with various significant differences as compared to means of control in the three seasons. A similar trend was also obtained regarding No. of fruits $/ \mathrm{m}$, fruit weight, length and diameter, fruit volume, yield and flesh thickness. The percent of TSS was increased in fruits of fertilized trees, while that of acidity was decreased in most cases of the three seasons. So, TSS/acidity ratio was often higher in fruits of treated trees than that in fruits of untreated ones. Moreover, vitamin $\mathrm{C}$ content in fruit flesh and fruit firmness were also increased. The leaf content of $\mathrm{N}, \mathrm{P}, \mathrm{K}, \mathrm{Ca}$ and $\mathrm{Mg}$ was markedly improved as a result of applying fertilization combinations used in such investigation. However, the mastery in most previous parameters was for a combination of $100 \%$ compost $+50 \%$ feldspar $+50 \%$ rock phosphate + bio-fertilizers mixture, which gave the best values in most cases of the three seasons.

Accordingly, it can be recommended to fertilize the 6-years-old irrigated trees of guava cv. "Etmani" with the combination of $100 \%$ compost $+50 \%$ feldspar $+50 \%$ rock phosphate plus mixture of biofertilizers to attain the highest growth, yield and fruit quality from commercial point of view.
\end{abstract}

\section{INTRODUCTION}

Guava (Psidium guajava L.) is one of the most lovely popular fruits, as it characterized by its high nutritive value and considered a rich source of vitamin $\mathrm{C}$ and several nutrients useful for human health and nutrition. Besides eating it in fresh form, it can be consume as food products, such as jam, jelly or juice (Wilson, 1980). Guava exceeds the majority of tropical and subtropical fruit trees in adaptability, productivity and tolerance to a wide range of soil and climatic conditions. It is grown now in more than 60 countries of the 
world. Owing to its high nutraceutical values, there is a growing consumers preference, and that resulting in expansion of area in many countries (Mitra et al., 2010)

Increasing demand for safe and healthy food makes organic farming very urgent, especially that guava is eaten fresh immediately after harvest. The organic farming system greatly relies on application of animal waste or farm yard manure, compost, crop rotation, crop residue, green manure, oil cakes, vermicompost and biofertilizers (Willer and Kilcher, 2010). This truth was documented by Ram et al., (2007) who reported that maximum fruit number and yield of guava cv. Allahabad Safeda were recorded consistently for 2 years in trees supplemented with $20 \mathrm{~kg} F Y M$ and inoculated with Azotabacter. Fruit content of TSS and vitamin $\mathrm{C}$, as well as leaf nutrients $(\mathrm{N}$, $\mathrm{P}, \mathrm{K}, \mathrm{Ca}, \mathrm{S}, \mathrm{Zn}, \mathrm{Cu}, \mathrm{Mn}$ and $\mathrm{Fe}$ ) levels were optimum with the application of different organic treatments. Likewise, Baksh et al., (2008) indicated that the maximum increment in growth of guava cv. Sardar trees (plant height, spread and trunk girth), improvement in yield and yield attributing characters (fruit set, retention and individual fruit weight) and quality of fruit (TSS, vitamin C, reducing and non-reducing sugars) were attained by $100 \% \mathrm{NPK}+250 \mathrm{~g}$ phosphate solubilizing bacteria (PSB) $+250 \mathrm{~g}$ Azotobacter treatment, which was at par with $75 \% \mathrm{NPK}+250 \mathrm{~g} \mathrm{PSB}+250 \mathrm{~g}$ Azotobacter one.

Similar observations were also postulated on various cultivars of guava by Dutta et al., (2009), Shalini et al., (2010), Rubee Lata et al., (2011), Goswami and Shant Lal Misra (2012), Trivedi et al., (2012), Akash Sharma Wali et al., (2013) and Meena et al., (2013) who found that application of 2/3 quantity of recommended dose of fertilizers, i.e. 500: $200: 500 \mathrm{~g} \mathrm{NPK}+25$ $\mathrm{kg} F Y M+250 \mathrm{~g}$ Azospirillum $+250 \mathrm{~g}$ Azotobacter in rejuvenated guava orchard cv. Sardar significantly increased number of fruits/ plant, yield/plant and yield/ha, as well as the leaf content of N, P and K.

Recently, Adak et al., (2014) and El-Taweel et al., (2005) stated that soils fertilized with vermicompost, biofertilizers and organic mulching showed improvement in growth and yield of guava as compared to NPK + FYM application. Moreover, Devi et al., (2014) declared that for cultivation of "Sardar" guava organically, application of FYM (26 kg/tree/year) + Azotobacter (100 g/tree) + phosphorus solubilizers $(100 \mathrm{~g} /$ tree $)+$ potash mobilizers (100 g/tree) in two splits (January and August) is the economically profitable treatment.

On olive cv. Picual, Gowda et al. (2011) noticed that feldspar at 3 $\mathrm{kg} /$ tree gave the highest vegetative growth, increased leaf pigments and mineral contents, higher values of fruit set, tree yield and fruit quality. Likely, El-Iraqy (2014) concluded that added olive pomace, compost, rock phosphate and feldspar alone or combined with nitropeine, phosphoreine and potaseine biofertilizers improved both vegetative growth, flowering, production and fruit quality parameters.

However, this work was set out to explore the enhancement effect of biofertilizers on increasing the beneficial returns of compost and mineral rocks on growth, fruit productivity and quality of guava fruits cv. "Etmani" trees under the ordinary surface irrigation system. 


\section{MATERIALS AND METHODS}

A field experiment was carried out in a private orchard at Qalyob district, Qalubia governorate, Egypt during the three consecutive seasons of 2012, 2013 and 2014 to examine the effect of compost, mineral rocks and biofertilizers in combinations at various levels on growth, productivity and quality of guava (Psidium guajava L.) fruits cv. "Etmani".

Therefore, uniform irrigated trees of 6-years-old planted on clay loem soil at a distance of $5 \times 5 \mathrm{~m}$, subjected to flood irrigation and received the useful agricultural practices needed for such plantation were selected well before each season to be similar as possible in their growth vigour, flowering time, fruit physical characteristics and time of maturity and devoted for this study.

The different sources of fertilization materials used in the current work at the recommended doses were as follows: Al-Obour compost (40 kg/tree), feldspar $(1.2 \mathrm{~kg} / \mathrm{tree})$ and rock phosphate $(1.3 \mathrm{~kg} / \mathrm{tree})$. The physical and chemical analysis of the soil and Al-Obour compost were determined and illustrated in Tables (1 and 2), while those of feldspar and rock phosphate are shown in Table (3).

Table (1): The mechanical, physical and chemical properties of the studied soil in the 3 seasons.

\begin{tabular}{|c|c|c|}
\hline Property & & Values \\
\hline \multirow{4}{*}{ Mechannical analysis } & Coarse sand & $7.41(\%)$ \\
\hline & Lime Sand & $23.71(\%)$ \\
\hline & Silt & $28.89(\%)$ \\
\hline & Clay & $30.72(\%)$ \\
\hline Texture (physical) & \multicolumn{2}{|c|}{ Clay loam } \\
\hline \multirow{6}{*}{ Chemical analysis } & $\mathrm{pH}$ & 7.62 \\
\hline & E.C. $(\mathrm{dSm}-1)$ & 3.1 \\
\hline & O.C. & 0.71 \\
\hline & O.M. & $1.24(\%)$ \\
\hline & T. N. & 0.17 \\
\hline & W.H.C. & 54.32 \\
\hline \multirow{6}{*}{ Anions and Cations (meq L-1) } & Bicarbonate $\left(\mathrm{HCO}_{3}^{-}\right)$ & 8.4 \\
\hline & Chloride $\left(\mathrm{Cl}^{-}\right)$ & 11.71 \\
\hline & Sulphate $\left(\mathrm{SO}_{4}{ }^{--}\right)$ & 16.43 \\
\hline & Calcium $\left(\mathrm{Ca}^{++}\right)$ & 8.53 \\
\hline & Magnesium $\left(\mathrm{Mg}^{++}\right)$ & 2.57 \\
\hline & Sodium $\left(\mathrm{Na}^{+}\right)$ & 22.93 \\
\hline
\end{tabular}

Soil analysis was done by: Soil, Water and Environment Res. Inst., ARC, Giza Egypt. 
Table (2): Physical and chemical analysis of Al-Obour compost used in the three seasons.

\begin{tabular}{|l|c|}
\hline \multicolumn{2}{|c|}{ Al-Obour compost } \\
\hline Character & Content \\
\hline Weight of $/ \mathrm{m}^{3}(\mathrm{~kg})$ & $500-550$ \\
\hline Humidity $\%)$ & $25-30$ \\
\hline $\mathrm{pH}(1-2.5)$ & $7.5-8.0$ \\
\hline Ec (1:5) & $3 .-4$ \\
\hline Water hold capacity & $250-300 \%$ \\
\hline Total nitrogen & $1-1.4 \%$ \\
\hline Organic matter & $34-38 \%$ \\
\hline Organic carbon & $19.8-22 \%$ \\
\hline C/N ratio & $1-14.2$ \\
\hline NaCl & $1.1-1.25 \%$ \\
\hline Total phosphorus & $0.5-0.75 \%$ \\
\hline Total potassium & $1.25-1.75 \%$ \\
\hline Fe (ppm) & $1500-1800$ \\
\hline $\mathrm{Mn}(\mathrm{ppm})$. & $25-50$ \\
\hline $\mathrm{Cu}(\mathrm{ppm})$ & $50-75$ \\
\hline $\mathrm{Zn}(\mathrm{ppm})$ & $150-225$ \\
\hline
\end{tabular}

The used compost manufactured from residues and free from heavy minerals and pollution. Compost analysis by: Producer Company.

Table (3): The chemical analysis of feldspar and rock phosphate used in the three seasons.

\begin{tabular}{|l|c|c|c|c|}
\hline \multirow{2}{*}{ Component (\%) } & \multicolumn{2}{|c|}{ Feldspar } & \multicolumn{2}{c|}{ Rock phosphate } \\
\cline { 2 - 5 } & From & To & From & To \\
\hline $\mathrm{SiO}_{2}$ & 68.56 & 70.23 & 10.60 & 12.78 \\
\hline $\mathrm{TiO}_{2}$ & 0.02 & 0.04 & 0.02 & 0.03 \\
\hline $\mathrm{Al}_{2} \mathrm{O}_{3}$ & 13.23 & 16.25 & 0.35 & 0.65 \\
\hline $\mathrm{Fe}_{2} \mathrm{O}_{3}$ & 0.17 & 0.40 & 1.12 & 1.35 \\
\hline $\mathrm{MnO}$ & 0.02 & 0.06 & 0.07 & 0.08 \\
\hline $\mathrm{Mg} \mathrm{O}$ & 0.03 & 0.05 & 0.33 & 0.61 \\
\hline $\mathrm{CaO}$ & 0.26 & 0.47 & 44.12 & 48.63 \\
\hline $\mathrm{Na}_{2} \mathrm{O}$ & 2.25 & 3.69 & 0.18 & 1.12 \\
\hline $\mathrm{K}_{2} \mathrm{O}$ & 6.20 & 8.12 & 0.03 & 0.05 \\
\hline $\mathrm{P}_{2} \mathrm{O}_{5}$ & 0.02 & 0.03 & 20.00 & 22.00 \\
\hline $\mathrm{SO}_{3}(\%)$ & - & - & 0.32 & 1.98 \\
\hline
\end{tabular}

Mineral rock analysis by: Producer Company.

However, the abovenamed materials were applied together at different proportion (100, 75, 50,25 and $0 \%$ for each) in the presence of nitropeine (a mixture of $\mathrm{N}$-fixing bacteria) at $120 \mathrm{~g} /$ tree, phosphoreine (a mixture of $\mathrm{p}$ solubilizing bacteria) at $25 \mathrm{~g} /$ tree and potasseine (a commercial product that contains $30 \% \mathrm{~K}_{2} \mathrm{O}$ and $\left.8 \% \mathrm{P}_{2} \mathrm{O}_{5}\right)$ at $134 \mathrm{~cm} /$ tree to formalize the following 12 combinations:

1. Control $\left(25 \mathrm{~kg}\right.$ as $\mathrm{FYM}+1.5 \mathrm{~kg}$ as $\mathrm{SO}_{4}(\mathrm{NH})_{2}+1 \mathrm{~kg}$ as $\mathrm{CA} \mathrm{H}_{2} \mathrm{P}_{2} \mathrm{O}_{5}$ and $1 \mathrm{~kg}$ as $\mathrm{k}_{2} \mathrm{SO}_{4} /$ tree). 
2. $100 \%$ Compost $+100 \%$ Feldspar $+100 \%$ Rock-P + Biofertilizers $\left(T_{1}\right)$

3. $100 \%$ Compost $+75 \%$ Feldspar $+75 \%$ Rock-P + Biofertilizers $\left(\mathrm{T}_{2}\right)$

4. $100 \%$ Compost $+50 \%$ Feldspar $+50 \%$ Rock-P + Biofertilizers $\left(\mathrm{T}_{3}\right)$

5. $100 \%$ Compost $+25 \%$ Feldspar $+25 \%$ Rock-P + Biofertilizers $\left(\mathrm{T}_{4}\right)$

6. $75 \%$ Compost $+100 \%$ Feldspar $+100 \%$ Rock-P + Biofertilizers $\left(T_{5}\right)$

7. $75 \%$ Compost $+75 \%$ Feldspar $+75 \%$ Rock- $P+$ Biofertilizers $\left(\mathrm{T}_{6}\right)$

8. $75 \%$ Compost $+50 \%$ Feldspar $+50 \%$ Rock- $P+$ Biofertilizers $\left(T_{7}\right)$

9. $75 \%$ Compost $+25 \%$ Feldspar $+25 \%$ Rock-P + Biofertilizers $\left(\mathrm{T}_{8}\right)$

10. $50 \%$ Compost $+100 \%$ Feldspar $+100 \%$ Rock-P + Biofertilizers $\left(T_{9}\right)$

$11.50 \%$ Compost $+75 \%$ Feldspar $+75 \%$ Rock- $P+$ Biofertilizers $\left(T_{10}\right)$

12. $50 \%$ Compost $+50 \%$ Feldspar $+50 \%$ Rock- $P+$ Biofertilizers $\left(T_{11}\right)$

13. $50 \%$ Compost $+25 \%$ Feldspar $+25 \%$ Rock- $P+$ Biofertilizers $\left(T_{12}\right)$

On mid of December for each season, the total amount of biofertilizers was mixed well with that of compost (specified for each treatment), as well as the total one of rock phosphate (also assigned for each treatment) were added to the soil at a depth of $20-25 \mathrm{~cm}$ where the most feeder roots of guava are present, in circled narrow trenches at $1 \mathrm{~m}$ away around trunk of each tree just before irrigation, and then properly covered with soil. Whereas, the feldspar doses were divided into two equal batches, as the first one applied on mid of December (with compost and biofertilizers) and the second one on June, $1^{\text {st }}$. The layout of the experiment in the three seasons was a complete randomized design, replicated thrice as each one contained 1 tree (Mead et al., 1993).

Data recorded:

At the proper time, data were registered as follows:

* Vegetative and flowering growth:

Shoot length $(\mathrm{cm})$, number of leaves $/ \mathrm{lm}$, leaf area $\left(\mathrm{cm}^{2}\right)$ using planimeter and number of flower buds/lm.

${ }^{*}$ Fruit characteristics and yield:

Number of fruits/lm, length and diameter of fruit $(\mathrm{cm})$, fruit size $\left(\mathrm{cm}^{3}\right)$, fruit weight $(\mathrm{g})$, flesh thickness $(\mathrm{cm})$, fruit firmness $\left(\mathrm{g} / \mathrm{cm}^{2}\right)$ and yield $(\mathrm{kg} / \mathrm{tree})$.

* Fruit chemical properties:

- Total soluble solids (TSS \%) were determined by a 'bbe refractometer using the method of A.O.A.C. (1995).

- Total acidity (\%) was measured by titration method described by A.O.A.C. (1975).

- TSS/acidity was calculated as a ratio.

- Vitamin C (ascorbic acid) was evaluated by the method of Horwitz (1970) as $\mathrm{mg} / 100 \mathrm{~g}$ fruit flesh.

- Leaf content of minerals.

In dry leaf samples taken from the middle part of the shoot, the percentages of nitrogen (A.O.A.C., 1995), phosphorus (Wide et al., 1985), potassium (by flame photometer set as indicated by Jackson, (1973) and calcium and magnesium (Dewis and Freitas, 1970) were assessed.

* Statistical analysis:

Data were then tabulated and statistically analyzed according to SAS Institute program (1994) using Duncan's Multiple Range Test (Duncan, 1955) for elucidating the significancy between the means of various treatments. 


\section{RESULTS AND DISCUSSION}

\section{Effect of fertilization treatments on:}

\section{1- Vegetative growth and flower bud number:}

It is clear from data averaged in Table (4) that means of shoot length $(\mathrm{cm})$, number of leaves $/ \mathrm{m}$ and leaf area $\left(\mathrm{cm}^{2}\right)$ were mostly improved in response to the different fertilization treatments employed in this study with various significant differences compared to control in the three studied seasons. However, the superiority was for $100 \%$ compost $+50 \%$ feldspar + $50 \%$ rock phosphate + biofertilizer mixture combined treatments, which gave the highest records in most cases of the three seasons and followed by a combination of $75 \%$ compost $+50 \%$ feldspar $+50 \%$ rock phosphate + biofertilizer mixture that recorded means closely near to those of super treatment with non-significant differences among them, especially in the traits of number of leaves $/ \mathrm{m}$ and leaf area. A similar trend was also gained regarding the number of flower buds $/ \mathrm{m}$ character, as the means of such parameter were increased in the three seasons to the maximal values by the super treatment mentioned above and followed by the same combination also indicated before.

This improvement in vegetative growth of guava trees may by attributed to the synergistic effect of organic matter compost which can improve the soil physical and chemical properties, increase water holding capacity, nutrient availability, soil organic matter content, cation exchange capacity and fertility, as well as EC and $\mathrm{pH}$ of the soil (Willer and Kilcher, 2011), feldspar which improve soil aggregation, structure, permeability and infiltration corresponding with the reduction of $\mathrm{pH}, \mathrm{EC}, \mathrm{SAR}$ and $\mathrm{Na} / \mathrm{Ca}$ ratio which leads finally to create better soil conditions (Meena et al., 2013) and rock phosphate which slowly supplies plants which mono-and di-phosphate, the most absorbing forms by plants (Adak et al., 2014). In this concern, Manning (2010) mentioned that feldspar as a source of $\mathrm{K}^{+}$reduced the osmotic pressure and increased water uptake which due to $\mathrm{K}^{+}$influx in soil solution, consequently increased the availability of some macro and micronutrients, and this may indicate its role in promoting and enhancing the metabolic process and regulating water balance. Moreover, the positive effects of feldspar not only ascribed to the multi-benefits of $\mathrm{K}^{+}$ions, but also to its containing $70.23 \% \mathrm{SiO}_{2}, 16.25 \% \mathrm{Al}_{2} \mathrm{O}_{3}, 8.12 \% \mathrm{~K}_{2} \mathrm{O}_{3} .69 \% \mathrm{Na}_{2} \mathrm{O}$ and traces of other elements such as $\mathrm{Fe}, \mathrm{Mg}, \mathrm{P}, \mathrm{Mn}, \mathrm{Ca}$ and $\mathrm{Ti}$ (as indicated in Table, (3).

In addition, biofertilizers (such as those used in the current work) in the presence of organic compost, feldspar and rock phosphate play a vital role in decomposing and solubilizing $\mathrm{N}, \mathrm{P}, \mathrm{K}, \mathrm{Ca}$ and other minerals to be available for plants. In this connection, Devi et al., (2014) suggested that microorganisms of biofertilizers may fix atmospheric $\mathrm{N}_{2}$, secrete some growth promoting factors, e.g. gibberellin, cytokinin- like substances, auxins and some vitamins and may release $\mathrm{K}$ and $\mathrm{P} 2 \mathrm{O} 5$ ions from rocks and organic materials to be available for plants. Subba-Rao (1993) declared that Azotobacter bacteria synthesize antifungal antibiotics which allow additional advantage for the use in field of crop production. 
J. Plant Production, Mansoura Univ., Vol. 6 (7), July, 2015

$4-$

1105 
The previous results go in line with those postulated on guava by Ram et al., (2007), Baksh et al., (2008), Dutta et al., (2009), Rubee Lata et al., (2011), Trivedi et al., (2012) and Akash Sharma Wali et al., (2013) who decided that the highest soil and leaf $\mathrm{N}, \mathrm{P}, \mathrm{Ca}$ and $\mathrm{Mg}$ contents was obtained with the treatment comprising Azotobacter $+25 \%$ of N/tree through FYM + $75 \%$ of $\mathrm{N} /$ tree through inorganic fertilizer, whereas the highest soil and leaf $\mathrm{K}$ contents was obtained with the application of Azotobacter $+50 \%$ of $\mathrm{N} /$ tree through $\mathrm{FYM}+50 \%$ of $\mathrm{N} /$ tree through inorganic fertilizer.

\section{2- Fruit characteristics and yield:}

Parallel observations to those of vegetative growth were also obtained in the matter of fruit characteristics and yield of irrigated guava trees, where data listed in Table (5) exhibited that No. of fruits/longitudinal meter, fruit weight $(\mathrm{g})$, yield $(\mathrm{kg} / \mathrm{tree})$, fruit length and diameter $(\mathrm{cm})$, fruit volume $(\mathrm{cm} 3)$ and flesh thickness $(\mathrm{cm})$ were generally improved as a result of applying the various fertilization treatments used in this trial with the mastery of $100 \%$ compost $+50 \%$ feldspar $+50 \%$ rock phosphate + bio-fert. combined treatment, which recorded the utmost high values over control and other combinations in most instances of the 3 studied seasons, but the combination of $75 \%$ compost $+100 \%$ feldspar $+100 \%$ rock phosphate + bio-fert. occupied the second category in this concern.

These results could be interpreted and discussed as done before in case of vegetative growth and No. of flower buds $/ \mathrm{m}$. Similarly were those findings revealed on guava by Shalini et al., (2010), Goswami and Sant Lal Misra (2012), Meena et al., (2013) and Adak et al., (2014). In this concern, Devi et al., (2014) found that the higher fruit weight and size of guava cv. Sardar were obtained by application of either neem cake $(9 \mathrm{~kg} / \mathrm{plant} / \mathrm{y})$ or vermicompost $(19 \mathrm{~kg} / \mathrm{plant} / \mathrm{y})+$ Azotobacter $(100 \mathrm{~g} / \mathrm{plant} / \mathrm{y})+P$-solubilizer $(100 \mathrm{~g} /$ plant/y) + K-mobilizer (100 g/plant/y). Maximum No. of fruits/plant was produced from plants fertilized with FYM $(26 \mathrm{~kg} / \mathrm{plant} / \mathrm{y})+$ Azotobacter + Psolubilizer $+\mathrm{K}$-mobilizer combined treatment, which also caused maximum yield compared to control. 
Table (5): Effect of fertilization treatments on yield and fruit characteristics of (Psidium guajava L.) "Etmani" cv. tree under ordinary irrigation system during 2012, 2013 and 2014 seasons.

\begin{tabular}{|c|c|c|c|c|c|c|c|}
\hline Treatments & $\begin{array}{l}\text { No. } \\
\text { fruits } \\
\text { per Im }\end{array}$ & $\begin{array}{c}\text { Fruit } \\
\text { weight } \\
\text { (g) }\end{array}$ & $\begin{array}{c}\text { Yield } \\
\text { (kg/tree) })\end{array}$ & $\begin{array}{c}\text { Fruit } \\
\text { length } \\
(\mathrm{cm})\end{array}$ & \begin{tabular}{|c|} 
Fruit \\
diameter \\
(cm)
\end{tabular} & \begin{tabular}{|c|}
$\begin{array}{c}\text { Fruit } \\
\text { volume } \\
\left(\mathrm{cm}^{3}\right)\end{array}$ \\
\end{tabular} & \begin{tabular}{|c|} 
Flesh \\
thickness \\
(cm)
\end{tabular} \\
\hline & \multicolumn{7}{|c|}{ First season: 2012} \\
\hline Control & $30.63 b-d$ & $48.97 d$ & $30.00 \mathrm{e}$ & $3.67 \mathrm{bc}$ & $2.77 \mathrm{e}$ & $42.67 \mathrm{~g}$ & $1.43 c$ \\
\hline $100 \% C+100 \% \mathrm{~K}+100 \% \mathrm{P}+$ Bio-F. & $31.25 \mathrm{bc}$ & $49.27 d$ & $30.67 b-d$ & $3.67 \mathrm{bc}$ & $3.07 \mathrm{bc}$ & $49.00 f$ & $1.40 c$ \\
\hline $100 \% \mathrm{C}+75 \% \mathrm{~K}+75 \% \mathrm{P}+\mathrm{Bio}-\mathrm{F}$. & $30.74 b-d$ & $51.45 c$ & $33.33 b-d$ & $3.70 \mathrm{bc}$ & $2.97 \mathrm{~cd}$ & $51.00 c-f$ & $1.30 \mathrm{~d}$ \\
\hline $100 \% \mathrm{C}+50 \% \mathrm{~K}+50 \% \mathrm{P}+\mathrm{Bio}-\mathrm{F}$. & $34.56 a$ & $53.16 \mathrm{ab}$ & $40.00 \mathrm{a}$ & $5.27 a$ & $3.37 a$ & $55.00 \mathrm{a}$ & $1.63 a$ \\
\hline $100 \% \mathrm{C}+25 \% \mathrm{~K}+25 \% \mathrm{P}+\mathrm{Bio}-\mathrm{F}$. & 29.60de & $51.15 c$ & $28.33 d$ & $4.10 \mathrm{bc}$ & $3.00 \mathrm{~cd}$ & $51.00 c-f$ & $1.43 \mathrm{c}$ \\
\hline $75 \% \mathrm{C}+100 \% \mathrm{~K}+100 \% \mathrm{P}+$ Bio-F. & $31.89 \mathrm{~b}$ & $53.71 \mathrm{a}$ & $39.00 a$ & $5.17 a$ & $2.93 c-e$ & $54.33 a b$ & $1.57 a b$ \\
\hline $75 \% \mathrm{C}+75 \% \mathrm{~K}+75 \% \mathrm{P}+$ Bio-F. & $31.79 \mathrm{~b}$ & $51.86 \mathrm{bc}$ & $31.67 b-d$ & $4.27 \mathrm{~b}$ & $2.93 c-e$ & $50.00 d-f$ & $1.60 a b$ \\
\hline $75 \% \mathrm{C}+50 \% \mathrm{~K}+50 \% \mathrm{P}+$ Bio-F. & $30.19 c-e$ & $52.05 b c$ & $31.33 b-d$ & $4.50 \mathrm{ab}$ & $3.10 \mathrm{bc}$ & $52.00 \mathrm{~b}-\mathrm{e}$ & $1.43 c$ \\
\hline $75 \% C+25 \% \mathrm{~K}+25 \% \mathrm{P}+$ Bio-F. & 29.62de & $52.06 \mathrm{bc}$ & $35.67 a b$ & $3.70 \mathrm{bc}$ & $3.03 b-d$ & $53.33 a-c$ & $1.60 \mathrm{ab}$ \\
\hline $50 \% \mathrm{C}+100 \% \mathrm{~K}+100 \% \mathrm{P}+$ Bio-F. & $30.24 c-e$ & $51.38 \mathrm{c}$ & $33.67 \mathrm{bc}$ & $3.33 c$ & $2.87 \mathrm{de}$ & $49.33 f$ & $1.60 \mathrm{ab}$ \\
\hline $50 \% \mathrm{C}+75 \% \mathrm{~K}+75 \% \mathrm{P}+$ Bio-F. & $27.91 \mathrm{f}$ & $51.94 \mathrm{bc}$ & $29.67 \mathrm{~cd}$ & $4.37 \mathrm{~b}$ & $3.07 \mathrm{bc}$ & $52.33 b-d$ & $1.57 a b$ \\
\hline $50 \% \mathrm{C}+50 \% \mathrm{~K}+50 \% \mathrm{P}+$ Bio-F. & $30.85 b-d$ & $51.98 \mathrm{bc}$ & $31.33 b-d$ & $3.73 b c$ & $3.10 \mathrm{bc}$ & 49.67ef & $1.53 a b$ \\
\hline \multirow[t]{2}{*}{$50 \% \mathrm{C}+25 \% \mathrm{~K}+25 \% \mathrm{P}+$ Bio-F. } & $28.86 e f$ & $52.58 \mathrm{a}-\mathrm{c}$ & $32.33 b-d$ & $3.73 b c$ & $3.20 \mathrm{~b}$ & $53.00 \mathrm{a}-\mathrm{c}$ & $1.50 \mathrm{bc}$ \\
\hline & \multicolumn{7}{|c|}{ Second season: 2013} \\
\hline Control & $25.97 i$ & $51.32 \mathrm{e}$ & $31.67 \mathrm{c}$ & $3.80 \mathrm{bc}$ & $2.83 \mathrm{c}$ & $43.33 f$ & $1.53 \mathrm{~cd}$ \\
\hline $100 \% \mathrm{C}+100 \% \mathrm{~K}+100 \% \mathrm{P}+$ Bio-F. & $29.97 \mathrm{~g}$ & $52.18 \mathrm{c}-\mathrm{e}$ & $35.67 \mathrm{c}$ & $3.90 \mathrm{bc}$ & $3.20 \mathrm{ab}$ & $51.00 \mathrm{e}$ & $1.50 \mathrm{~cd}$ \\
\hline $100 \% \mathrm{C}+75 \% \mathrm{~K}+75 \% \mathrm{P}+$ Bio-F. & $33.83 \mathrm{~cd}$ & $52.51 \mathrm{~b}-\mathrm{d}$ & $35.00 \mathrm{c}$ & $3.70 \mathrm{bc}$ & $3.03 \mathrm{bc}$ & 51.33de & $1.43 d$ \\
\hline $100 \% \mathrm{C}+50 \% \mathrm{~K}+50 \% \mathrm{P}+$ Bio-F. & $34.82 \mathrm{a}$ & $54.78 \mathrm{a}$ & $45.00 \mathrm{a}$ & $5.37 a$ & $3.37 a$ & $55.67 a$ & $1.70 \mathrm{a}$ \\
\hline $100 \% C+25 \% K+25 \% P+$ Bio-F. & $29.33 \mathrm{~h}$ & $52.50 \mathrm{~b}-\mathrm{d}$ & $34.00 \mathrm{c}$ & $4.23 \mathrm{bc}$ & $3.13 a b$ & $53.00 \mathrm{~b}-\mathrm{e}$ & $1.57 \mathrm{bc}$ \\
\hline $75 \% \mathrm{C}+100 \% \mathrm{~K}+100 \% \mathrm{P}+$ Bio-F. & $34.10 \mathrm{bc}$ & $53.33 b$ & $41.67 a b$ & $5.27 a$ & $3.33 a$ & $55.00 \mathrm{ab}$ & $1.60 \mathrm{a}-\mathrm{c}$ \\
\hline $75 \%$ C + $75 \%$ K + $75 \%$ P + Bio-F. & $32.76 \mathrm{e}$ & $52.62 b-d$ & $35.00 \mathrm{c}$ & $4.47 a-c$ & $3.07 \mathrm{~b}$ & $51.00 \mathrm{e}$ & $1.60 \mathrm{a}-\mathrm{c}$ \\
\hline $75 \% \mathrm{C}+50 \% \mathrm{~K}+50 \% \mathrm{P}+\mathrm{Bio}-\mathrm{F}$. & $34.46 \mathrm{ab}$ & $53.07 \mathrm{bc}$ & $34.67 \mathrm{c}$ & $4.60 \mathrm{ab}$ & $3.20 \mathrm{ab}$ & $53.67 a-c$ & $1.53 \mathrm{~cd}$ \\
\hline $75 \% C+25 \% \mathrm{~K}+25 \% \mathrm{P}+$ Bio-F. & $31.57 f$ & $53.03 b-d$ & $40.00 \mathrm{~b}$ & $3.83 \mathrm{bc}$ & $3.17 a b$ & $53.33 b-d$ & $1.60 \mathrm{a}-\mathrm{c}$ \\
\hline $50 \% \mathrm{C}+100 \% \mathrm{~K}+100 \% \mathrm{P}+\mathrm{Bio}-\mathrm{F}$. & $32.02 f$ & $52.78 b-d$ & $35.00 \mathrm{c}$ & $3.53 \mathrm{c}$ & $3.07 \mathrm{~b}$ & $51.67 c-e$ & $1.67 a b$ \\
\hline $50 \% \mathrm{C}+75 \% \mathrm{~K}+75 \% \mathrm{P}+\mathrm{Bio}-\mathrm{F}$. & $31.87 f$ & $52.98 b-d$ & $33.33 \mathrm{c}$ & $4.60 \mathrm{ab}$ & $3.17 a b$ & $53.67 a-c$ & $1.67 a b$ \\
\hline $50 \% \mathrm{C}+50 \% \mathrm{~K}+50 \% \mathrm{P}+\mathrm{Bio}-\mathrm{F}$. & $33.37 d$ & $52.83 b-d$ & $35.67 \mathrm{c}$ & $4.03 \mathrm{bc}$ & $3.17 a b$ & $51.67 c-e$ & $1.57 \mathrm{bc}$ \\
\hline \multirow[t]{2}{*}{$50 \% \mathrm{C}+25 \% \mathrm{~K}+25 \% \mathrm{P}+$ Bio-F. } & $31.89 f$ & $51.95 \mathrm{de}$ & $35.00 \mathrm{c}$ & $3.73 b c$ & $3.03 \mathrm{bc}$ & $54.67 a-c$ & $1.70 \mathrm{a}$ \\
\hline & \multicolumn{7}{|c|}{ Third season: 2014} \\
\hline Control & $34.53 d-f$ & $51.93 f$ & $34.00 \mathrm{~d}$ & $3.97 b-e$ & $2.83 \mathrm{e}$ & $44.00 \mathrm{~h}$ & $1.63 a-c$ \\
\hline $100 \% \mathrm{C}+100 \% \mathrm{~K}+100 \% \mathrm{P}+$ Bio-F. & $31.42 \mathrm{~g}$ & $52.15 \mathrm{ef}$ & $40.00 \mathrm{c}$ & $3.93 c-e$ & $3.17 \mathrm{bc}$ & $51.00 \mathrm{fg}$ & $1.43 d$ \\
\hline $100 \% \mathrm{C}+75 \% \mathrm{~K}+75 \% \mathrm{P}+\mathrm{Bio}-\mathrm{F}$. & $35.10 d-f$ & $53.80 \mathrm{~cd}$ & $38.33 \mathrm{~cd}$ & 3.87de & $3.10 \mathrm{~b}-\mathrm{d}$ & $53.00 d-f$ & $1.50 \mathrm{~cd}$ \\
\hline $100 \% \mathrm{C}+50 \% \mathrm{~K}+50 \% \mathrm{P}+$ Bio-F. & $40.10 \mathrm{a}$ & $58.23 a$ & $51.67 a$ & $5.70 \mathrm{a}$ & $3.67 a$ & $60.00 \mathrm{a}$ & $1.77 \mathrm{a}$ \\
\hline $100 \% \mathrm{C}+25 \% \mathrm{~K}+25 \% \mathrm{P}+$ Bio-F. & $30.70 \mathrm{~g}$ & $53.56 \mathrm{~cd}$ & $36.67 \mathrm{~cd}$ & $4.43 b-d$ & $3.13 b-d$ & $54.67 \mathrm{~cd}$ & $1.63 a-c$ \\
\hline $75 \% \mathrm{C}+100 \% \mathrm{~K}+100 \% \mathrm{P}+$ Bio-F. & $37.52 b$ & $56.11 \mathrm{~b}$ & $48.33 a b$ & $5.57 a$ & $3.60 \mathrm{a}$ & $57.33 \mathrm{~b}$ & $1.77 \mathrm{a}$ \\
\hline $75 \% \mathrm{C}+75 \% \mathrm{~K}+75 \% \mathrm{P}+\mathrm{Bio}-\mathrm{F}$ & $35.76 c-e$ & $53.50 \mathrm{~cd}$ & $38.33 \mathrm{~cd}$ & $4.67 \mathrm{~b}-\mathrm{d}$ & 3.00c-e & $51.67 \mathrm{e}-\mathrm{g}$ & $1.70 a b$ \\
\hline $75 \% \mathrm{C}+50 \% \mathrm{~K}+50 \% \mathrm{P}+$ Bio-F. & $36.93 \mathrm{bc}$ & $52.97 d-f$ & $37.33 \mathrm{~cd}$ & $4.80 \mathrm{~b}$ & $3.30 \mathrm{~b}$ & $53.33 c-e$ & $1.57 b-d$ \\
\hline $75 \% \mathrm{C}+25 \% \mathrm{~K}+25 \% \mathrm{P}+$ Bio-F. & $33.58 f$ & $52.90 \mathrm{~d}-\mathrm{f}$ & $45.00 \mathrm{~b}$ & $4.13 \mathrm{~b}-\mathrm{e}$ & 3.13b-d & $55.33 c$ & $1.70 \mathrm{ab}$ \\
\hline $50 \% \mathrm{C}+100 \% \mathrm{~K}+100 \% \mathrm{P}+\mathrm{Bio}-\mathrm{F}$. & $31.20 \mathrm{~g}$ & $52.75 d-f$ & $40.00 \mathrm{c}$ & $3.57 \mathrm{e}$ & 2.90de & $50.67 \mathrm{~g}$ & 1.70ab \\
\hline $50 \% \mathrm{C}+75 \% \mathrm{~K}+75 \% \mathrm{P}+\mathrm{Bio}-\mathrm{F}$ & $31.84 \mathrm{~g}$ & 53.03de & $36.67 \mathrm{~cd}$ & $4.77 \mathrm{bc}$ & $3.20 \mathrm{bc}$ & $55.33 \mathrm{c}$ & $1.67 a b$ \\
\hline $50 \% \mathrm{C}+50 \% \mathrm{~K}+50 \% \mathrm{P}+$ Bio-F. & $34.48 \mathrm{ef}$ & $53.26 \mathrm{~cd}$ & $39.00 \mathrm{c}$ & $4.03 \mathrm{~b}-\mathrm{e}$ & $3.20 \mathrm{bc}$ & $52.33 \mathrm{e}-\mathrm{g}$ & $1.60 \mathrm{bc}$ \\
\hline $50 \% \mathrm{C}+25 \% \mathrm{~K}+25 \% \mathrm{P}+$ Bio-F. & $36.26 b-d$ & $54.21 \mathrm{c}$ & $40.00 \mathrm{c}$ & $3.87 \mathrm{de}$ & $3.07 \mathrm{~b}-\mathrm{e}$ & $55.33 c$ & $1.67 a b$ \\
\hline
\end{tabular}

* C: Compost; K: Feldspar; P: Rock phosphate, Bio-F.: Nitrobeine, Phosphorene, Potassein and Im: Longitudinal meter.

* Means within a column having the same letters are not significantly different according to Duncan's Multiple Range Test at $5 \%$ level. 


\section{3- Chemical composition and firmness of fruits:}

According to data presented in Table (6), it is clear that TSS \% was increased in the fruits of fertilized trees with various significant levels relative to control in the three seasons, whereas acidity \% was reduced in most cases of the 3 seasons. However, the highest percent of TSS coupled with the minimest one of acidity was often achieved in the 3 seasons by the combination of $100 \%$ compost $+50 \%$ feldspar $+50 \%$ rock phosphate + bio fert. So, fruits resulted from such treatment were more delicious than those resulted from other treatments, especially that this treatment was also registered the highest ratio of TSS/acidity, which considered a real indicator for palatability of guava fruits.

As for content of vitamin C (mg/100 g fruit flesh) and fruit firmness $\left(\mathrm{g} / \mathrm{cm}^{2}\right)$, they were also increased with few exceptions due to different fertilization treatments used in this work compared to control in the 3 studied seasons, although the variations between values of vitamin $\mathrm{C}$ content under various treatments were greatly narrow during the 3 seasons.

In general the prevalence in most aforementioned characters was found due to $100 \%$ compost $+50 \%$ feldspar $+50 \%$ rock phosphate + biofertilizers combination, which mostly scored the best averages, especially in the $1^{\text {st }}$ and $2^{\text {nd }}$ seasons. This may be reasonable because such combination established the best vegetative growth during the course of each season, and that positively reflected on fruit productivity and quality. These gains, however conform with those detected on guava cultivars by Ram et al., (2007), Baksh et al., (2008), Dutta et al., (2009), Goswami and Shant Lal Misra (2012) and Trivedi et al., (2012) who pointed out that cv. Sardar was more responding to organic manure and biofertilizer, so recorded higher plant height and spread and $\mathrm{N}$ uptake than cv. Allahabad Safeda, while Allahabad Safeda registered higher TSS and vitamin C content. On olive cv. Picual, Gowda et al., (2011) noticed that feldspar at $3 \mathrm{~kg} /$ tree gave higher fruit set, yield, quality and oil content. 
Table (6): Effect of fertilization treatments on chemical composition and firmness of (Psidium guajava L.) "Etmani" cv. fruits under ordinary irrigation system during 2012, 2013 and 2014 seasons

\begin{tabular}{|c|c|c|c|c|c|}
\hline Treatments & TSS (\%) & $\begin{array}{c}\text { Acidity } \\
\text { (\%) }\end{array}$ & $\begin{array}{l}\text { TSS/ } \\
\text { acidity } \\
\text { ratio }\end{array}$ & $\begin{array}{c}\text { Vitamin } \\
\mathrm{C} \\
(\mathrm{mg} / 100 \mathrm{~g} \\
\text { f.f. })\end{array}$ & $\begin{array}{c}\text { Fruit } \\
\text { firmness } \\
\left(\mathrm{g} / \mathrm{cm}^{2}\right)\end{array}$ \\
\hline & \multicolumn{5}{|c|}{ First season: 2012} \\
\hline Control & $9.30 \mathrm{~d}$ & $0.533 a$ & $17.45 \mathrm{e}$ & $39.90 \mathrm{~b}$ & $104.00 f$ \\
\hline $100 \% C+100 \% K+100 \% P+$ Bio-F. & $9.40 \mathrm{~d}$ & $0.467 \mathrm{ab}$ & $20.13 \mathrm{~cd}$ & $40.13 a$ & 112.0de \\
\hline 100 \% C + 75 \% K + 75 \% P + Bio-F. & $9.27 d$ & $0.467 a b$ & $19.85 d$ & $40.13 a$ & $107.0 \mathrm{ef}$ \\
\hline $100 \% \mathrm{C}+50 \% \mathrm{~K}+50 \% \mathrm{P}+\mathrm{Bio}-\mathrm{F}$. & $10.20 \mathrm{a}$ & $0.333 \mathrm{c}$ & $30.63 a$ & $40.17 a$ & $128.3 a$ \\
\hline $100 \% \mathrm{C}+25 \% \mathrm{~K}+25 \% \mathrm{P}+\mathrm{Bio}-\mathrm{F}$. & $9.23 d$ & $0.433 a b$ & $21.32 \mathrm{~cd}$ & $39.87 \mathrm{~b}$ & $126.7 \mathrm{a}$ \\
\hline $75 \% \mathrm{C}+100 \% \mathrm{~K}+100 \% \mathrm{P}+\mathrm{Bio}-\mathrm{F}$. & $10.00 \mathrm{~b}$ & $0.367 \mathrm{bc}$ & $27.25 a b$ & $40.07 a b$ & $128.3 a$ \\
\hline $75 \% \mathrm{C}+75 \% \mathrm{~K}+75 \% \mathrm{P}+\mathrm{Bio}-\mathrm{F}$ & $9.40 \mathrm{~d}$ & $0.467 a b$ & $20.13 \mathrm{~cd}$ & $39.97 a b$ & $118.3 \mathrm{c}$ \\
\hline $75 \% C+50 \% \mathrm{~K}+50 \% \mathrm{P}+$ Bio-F. & $9.40 d$ & $0.333 c$ & $28.23 a$ & $40.07 a b$ & $115.0 \mathrm{~cd}$ \\
\hline $75 \% C+25 \% \mathrm{~K}+25 \% \mathrm{P}+$ Bio-F. & $9.27 d$ & $0.400 \mathrm{bc}$ & $23.18 \mathrm{bc}$ & $40.13 a$ & $125.0 \mathrm{ab}$ \\
\hline $50 \% \mathrm{C}+100 \% \mathrm{~K}+100 \% \mathrm{P}+\mathrm{Bio}-\mathrm{F}$. & $9.03 e$ & $0.300 \mathrm{c}$ & $30.10 \mathrm{a}$ & $39.97 a b$ & $120.0 \mathrm{bc}$ \\
\hline $50 \%$ C + $75 \%$ K + $75 \%$ P + Bio-F. & 9.30d & $0.333 \mathrm{c}$ & $27.93 a b$ & $40.07 a b$ & $118.3 \mathrm{c}$ \\
\hline $50 \% \mathrm{C}+50 \% \mathrm{~K}+50 \% \mathrm{P}+$ Bio-F. & 9.30d & $0.333 \mathrm{c}$ & $27.93 a b$ & $40.03 a b$ & 107.7 ef \\
\hline \multirow[t]{2}{*}{$50 \% \mathrm{C}+25 \% \mathrm{~K}+25 \% \mathrm{P}+$ Bio-F. } & $9.60 \mathrm{c}$ & $0.467 a b$ & $20.56 \mathrm{~cd}$ & $40.03 a b$ & 107.7 ef \\
\hline & \multicolumn{5}{|c|}{ Second season: 2013} \\
\hline Control & 9.33ef & $0.567 a$ & $16.46 \mathrm{e}$ & 39.40de & $3.3 \mathrm{~cd}$ \\
\hline $100 \% \mathrm{C}+100 \% \mathrm{~K}+100 \% \mathrm{P}+$ Bio-F. & $9.70 \mathrm{~b}$ & $0.400 c-e$ & $24.25 \mathrm{c}$ & $40.10 a-d$ & $.0 \mathrm{e}$ \\
\hline $100 \% \mathrm{C}+75 \% \mathrm{~K}+75 \% \mathrm{P}+\mathrm{Bio}-\mathrm{F}$. & 9.37d-f & $0.467 \mathrm{bc}$ & $20.06 \mathrm{~d}$ & $40.17 \mathrm{~b}-\mathrm{d}$ & 115.0de \\
\hline $100 \% \mathrm{C}+50 \% \mathrm{~K}+50 \% \mathrm{P}+$ Bio-F. & $10.23 a$ & $0.367 \mathrm{de}$ & $27.87 \mathrm{~b}$ & $40.47 \mathrm{a}$ & $35.0 \mathrm{a}$ \\
\hline $100 \% \mathrm{C}+25 \% \mathrm{~K}+25 \% \mathrm{P}+\mathrm{Bio}-\mathrm{F}$ & $9.40 c-f$ & $0.533 a b$ & $64 \mathrm{e}$ & $40.00 \mathrm{da}-\mathrm{c}$ & 133.3ab \\
\hline $75 \% C+100 \% \mathrm{~K}+100 \% \mathrm{P}+$ Bio-F. & $10.20 \mathrm{a}$ & & $63 a$ & 39.47de & $131.7 a b$ \\
\hline $75 \% \mathrm{C}+75 \% \mathrm{~K}+75 \% \mathrm{P}+\mathrm{Bio}-\mathrm{F}$. & $9.60 \mathrm{bc}$ & $33 \mathrm{~cd}$ & $22.17 \mathrm{c}$ & 10a-d & 133.3ab \\
\hline $75 \%$ C + $50 \% \mathrm{~K}+50 \% \mathrm{P}+$ Bio-F. & $9.40 c-f$ & $33 \mathrm{~cd}$ & $21.71 \mathrm{~cd}$ & $39.90 \mathrm{e}$ & $128.3 \mathrm{~b}$ \\
\hline $75 \%$ C + $25 \%$ K + $25 \%$ P + Bio-F. & $9.30 f$ & $0.433 \mathrm{~cd}$ & $21.48 \mathrm{c}$ & 40.37ab & $131.7 \mathrm{ab}$ \\
\hline $50 \% \mathrm{C}+100 \% \mathrm{~K}+100 \% \mathrm{P}+\mathrm{Bio}-\mathrm{F}$ & $9.57 \mathrm{~b}-\mathrm{d}$ & $0.367 \mathrm{de}$ & $26.08 \mathrm{~b}$ & $40.33 a-c$ & $131.7 a b$ \\
\hline$\% \mathrm{P}+\mathrm{Bio}-\mathrm{F}$ & $9.57 \mathrm{~b}-\mathrm{d}$ & $0.433 \mathrm{~cd}$ & $.10 \mathrm{c}$ & $40.40 \mathrm{a}-\mathrm{c}$ & $110.0 \mathrm{e}$ \\
\hline $50 \% \mathrm{C}+50 \% \mathrm{~K}+50 \% \mathrm{P}+$ Bio-F. & $9.53 b-e$ & $0.433 \mathrm{~cd}$ & $.01 \mathrm{c}$ & $40.17 a-d$ & $131.7 \mathrm{ab}$ \\
\hline \multirow[t]{2}{*}{$50 \% \mathrm{C}+25 \% \mathrm{~K}+25 \% \mathrm{P}+$ Bio-F. } & $9.70 \mathrm{~b}$ & $0.533 a b$ & $18.20 \mathrm{e}$ & $40.13 a-d$ & $121.7 \mathrm{c}$ \\
\hline & \multicolumn{5}{|c|}{ Third season: 2014} \\
\hline Control & $.53 \mathrm{~b}$ & $0.500 \mathrm{a}$ & $19.06 \mathrm{~d}$ & $39.90 \mathrm{e}$ & $120.0 \mathrm{~d}$ \\
\hline $100 \% \mathrm{C}+100 \% \mathrm{~K}+100 \% \mathrm{P}+\mathrm{Bio}-\mathrm{F}$. & $9.77 \mathrm{~b}$ & $0.467 a b$ & $20.92 \mathrm{c}$ & $40.40 \mathrm{a}$ & $3.3 d$ \\
\hline $100 \%$ C + $75 \% \mathrm{~K}+75 \% \mathrm{P}+$ Bio-F. & $9.53 b$ & $0.433 a-c$ & $22.00 \mathrm{c}$ & $40.37 a b$ & $113.3 d$ \\
\hline $100 \% \mathrm{C}+50 \% \mathrm{~K}+50 \% \mathrm{P}+$ Bio-F. & $11.07 a$ & $0.467 a b$ & $23.70 \mathrm{~b}$ & $40.30 \mathrm{a}-\mathrm{c}$ & $135.0 \mathrm{bc}$ \\
\hline $100 \% \mathrm{C}+25 \% \mathrm{~K}+25 \% \mathrm{P}+\mathrm{Bio}-\mathrm{F}$ & $9.47 \mathrm{~b}$ & $0.367 \mathrm{bc}$ & $25.80 \mathrm{~b}$ & 40.03de & $116.7 d$ \\
\hline $75 \% \mathrm{C}+100 \% \mathrm{~K}+100 \% \mathrm{P}+\mathrm{Bio}-\mathrm{F}$. & $10.83 a$ & $0.367 \mathrm{bc}$ & $29.51 a$ & $40.27 a-c$ & $145.0 \mathrm{a}$ \\
\hline $75 \%$ C + $75 \%$ K + $75 \%$ P + Bio-F. & $9.87 \mathrm{~b}$ & $0.500 \mathrm{a}$ & $19.74 \mathrm{e}$ & $40.13 b-d$ & $131.7 \mathrm{c}$ \\
\hline $75 \%$ C + $50 \% \mathrm{~K}+50 \% \mathrm{P}+$ Bio-F. & $9.60 \mathrm{~b}$ & $0.400 a-c$ & $24.00 \mathrm{~b}$ & $40.20 \mathrm{a}-\mathrm{d}$ & $135.0 \mathrm{bc}$ \\
\hline $75 \%$ C + $25 \% \mathrm{~K}+25 \% \mathrm{P}+\mathrm{Bio}-\mathrm{F}$ & $9.60 \mathrm{~b}$ & $0.467 a b$ & $20.56 \mathrm{~cd}$ & 40.37ab & $130.0 \mathrm{c}$ \\
\hline $50 \% \mathrm{C}+100 \% \mathrm{~K}+100 \% \mathrm{P}+\mathrm{Bio}-\mathrm{F}$ & $9.60 \mathrm{~b}$ & $0.333 \mathrm{c}$ & $28.83 a$ & $40.10 c-e$ & $140.0 a b$ \\
\hline 50 \% C + $75 \%$ K + 75 \% P + Bio-F. & $9.70 \mathrm{~b}$ & $0.333 \mathrm{c}$ & $29.13 a$ & $40.30 \mathrm{a}-\mathrm{c}$ & $131.7 \mathrm{c}$ \\
\hline $50 \% \mathrm{C}+50 \% \mathrm{~K}+50 \% \mathrm{P}+$ Bio-F. & $9.57 b$ & $0.400 a-c$ & $23.93 \mathrm{bc}$ & 40.37ab & $133.3 \mathrm{bc}$ \\
\hline $50 \% C+25 \% \mathrm{~K}+25 \% \mathrm{P}+$ Bio-F. & $9.80 \mathrm{~b}$ & $0.467 a b$ & $20.99 \mathrm{~cd}$ & 40.00de & $130.0 \mathrm{c}$ \\
\hline
\end{tabular}

${ }^{\star} \mathrm{C}$ : Compost; K: Feldspar; P: Rock phosphate, and Bio-F.: Nitrobeine, Phosphorene, Potassein.

* Means within a column having the same letters are not significantly different according to Duncan's Multiple Range Test at $5 \%$ level. 


\section{4- Mineral content of the leaves:}

It is obvious from data issued in Table (7) that the percentages of N, P, $\mathrm{K}, \mathrm{Ca}$ and $\mathrm{Mg}$ in the leaves of fertilized trees were increased as a result of dressing with the different fertilization treatments used in this investigation. The significancy among the means of various treatments were variable in most cases of the three seasons, but the dominance in the first and second seasons was ascribed to fertilizing with $100 \%$ compost $+50 \%$ feldspar +50 $\%$ rock phosphate + bio-fert. combined treatment, which followed by $75 \%$ compost $+100 \%$ feldspar $+100 \%$ rock phosphate + bio-fert. combined one. In the $3^{\text {rd }}$ season, the opposite was the right, where the latter combination preceded the former one.

This may indicate the role of microorganisms of biofertilizers in decomposing organic matter and mineral rocks, consequently mobilizing $\mathrm{K}_{2} \mathrm{O}$ and $\mathrm{P}_{2} \mathrm{O}_{5}$ plus other nutrients to be more available for plants (Subba-Rao, 1993). In this regard, Trivedi et al., (2012) reported that application of biocompost to Sardar and Allahabad Safeda guava soil recorded the maximum available $\mathrm{N}, \mathrm{K}_{2} \mathrm{O}$ and $\mathrm{P}_{2} \mathrm{O}_{5}$. incorporation of vermicompost resulted in the maximum $\mathrm{N}$ uptake and that of FYM resulted in the maximum $\mathrm{P}$ uptake and organic carbon content in the soil. Addition of biofertilizers recorded higher available $\mathrm{P}_{2} \mathrm{O}_{5}$ content in the soil. Similarly, were those results obtained by Ram et al., (2007) on guava cv. Allahabad Safeda, Rubee Lata et al., (2011) on guava cv. Red fleshed, Goswami and Shant Lal Misra (2012) on guava cv. Pant Prabhat, Akash Sharma Wali et al., (2013) on guava cv. Sardar and El-Iraqy (2014) on olive cv. Picual.

From the aforementioned results, it can be concluded that application of $100 \%$ of recommended compost dose $(40 \mathrm{~kg} / \mathrm{tree})+50 \%$ of recommended feldspar dose $(600 \mathrm{~g} /$ tree $)$ and $50 \%$ of recommended rock phosphate dose (650 $\mathrm{g} /$ tree) alongwith biofertilizers (nitropeine, phosphoreine and potasseine at 120 , 25 and $134 \mathrm{~g} /$ tree, respectively) to 6-years-old guava cv. "Etmani" tree under flood irrigation system may be one of the economic way for highly growth, production and fruit quality. 
Table (7): Effect of fertilization treatments on mineral content of (Psidium guajava L.) "Etmani" cv. leaves under ordinary irrigation system during 2012, 2013 and 2014 seasons

\begin{tabular}{|c|c|c|c|c|c|}
\hline Treatments & $\mathbf{N}(\%)$ & $\mathbf{P}(\%)$ & $\mathrm{K}(\%)$ & $\mathrm{Ca}(\%)$ & Mg (\%) \\
\hline & \multicolumn{5}{|c|}{ First season: 2012} \\
\hline Control & $1.522 \mathrm{~h}$ & $0.122 \mathrm{~g}$ & $1.392 i$ & $1.754 d$ & $0.410 \mathrm{gh}$ \\
\hline $100 \% C+100 \% \mathrm{~K}+100 \% \mathrm{P}+$ Bio-F. & $1.909 \mathrm{e}$ & $0.202 d$ & $1.543 \mathrm{c}$ & $1.764 d$ & $0.430 \mathrm{~g}$ \\
\hline $100 \% \mathrm{C}+75 \% \mathrm{~K}+75 \% \mathrm{P}+\mathrm{Bio}-\mathrm{F}$. & $1.632 \mathrm{~g}$ & $0.133 \mathrm{fg}$ & $1.546 \mathrm{bc}$ & $1.772 \mathrm{~cd}$ & $0.476 f$ \\
\hline $100 \% \mathrm{C}+50 \% \mathrm{~K}+50 \% \mathrm{P}+$ Bio-F. & $2.248 a$ & $0.386 a$ & $1.574 a$ & $1.726 \mathrm{e}$ & $0.411 \mathrm{gh}$ \\
\hline $100 \% \mathrm{C}+25 \% \mathrm{~K}+25 \% \mathrm{P}+$ Bio-F. & $1.531 \mathrm{~h}$ & $0.324 b$ & $1.521 d$ & $1.762 \mathrm{~d}$ & $0.414 \mathrm{gh}$ \\
\hline $75 \% C+100 \% \mathrm{~K}+100 \% \mathrm{P}+$ Bio-F. & $2.252 a$ & $0.142 f$ & $1.480 f$ & $1.757 d$ & $0.903 a$ \\
\hline $75 \%$ C + $75 \%$ K + $75 \%$ P + Bio-F. & $2.063 \mathrm{c}$ & $0.223 c$ & $1.474 \mathrm{fg}$ & $1.755 d$ & $0.825 \mathrm{c}$ \\
\hline $75 \% \mathrm{C}+50 \% \mathrm{~K}+50 \% \mathrm{P}+$ Bio-F. & 1.943de & $0.372 a$ & 1.513de & $1.728 \mathrm{e}$ & $0.425 \mathrm{gh}$ \\
\hline $75 \% C+25 \% K+25 \% P+$ Bio-F. & $1.563 \mathrm{~h}$ & $0.194 d$ & $1.457 \mathrm{gh}$ & $1.757 d$ & $0.506 \mathrm{e}$ \\
\hline $50 \% \mathrm{C}+100 \% \mathrm{~K}+100 \% \mathrm{P}+$ Bio-F. & $1.971 d$ & $0.125 f g$ & $1.563 a b$ & $1.762 d$ & $0.694 d$ \\
\hline $50 \% \mathrm{C}+75 \% \mathrm{~K}+75 \% \mathrm{P}+\mathrm{Bio}-\mathrm{F}$ & $1.550 \mathrm{~h}$ & $0.159 \mathrm{e}$ & $1.502 \mathrm{e}$ & $1.824 a$ & $0.494 \mathrm{ef}$ \\
\hline $50 \% \mathrm{C}+50 \% \mathrm{~K}+50 \% \mathrm{P}+$ Bio-F. & $1.850 f$ & $0.121 \mathrm{~g}$ & $1.332 \mathrm{j}$ & $1.792 b$ & $0.405 \mathrm{~h}$ \\
\hline \multirow[t]{2}{*}{$50 \% \mathrm{C}+25 \% \mathrm{~K}+25 \% \mathrm{P}+$ Bio-F. } & $2.192 b$ & $0.334 b$ & $1.442 \mathrm{~h}$ & $1.787 \mathrm{bc}$ & $0.725 \mathrm{c}$ \\
\hline & \multicolumn{5}{|c|}{ Second season: 2013} \\
\hline Control & $1.582 \mathrm{~h}$ & $0.188 \mathrm{~d}$ & $1.448 \mathrm{c}$ & $1.707 \mathrm{~g}$ & $0.431 \mathrm{f}$ \\
\hline $100 \% \mathrm{C}+100 \% \mathrm{~K}+100 \% \mathrm{P}+$ Bio-F. & $1.775 f$ & $0.204 d$ & $1.643 a b$ & $1.751 d$ & $0.505 \mathrm{e}$ \\
\hline $100 \% \mathrm{C}+75 \% \mathrm{~K}+75 \% \mathrm{P}+$ Bio-F. & $1.985 d$ & $0.226 \mathrm{~cd}$ & $1.679 a$ & $1.727 \mathrm{ef}$ & $0.361 \mathrm{~g}$ \\
\hline $100 \% \mathrm{C}+50 \% \mathrm{~K}+50 \% \mathrm{P}+$ Bio-F. & $2.282 a$ & $0.555 \mathrm{a}$ & $1.684 a$ & 1.736de & $0.794 a$ \\
\hline $100 \% \mathrm{C}+25 \% \mathrm{~K}+25 \% \mathrm{P}+$ Bio-F. & $2.279 a$ & $0.331 \mathrm{bc}$ & $1.634 a b$ & $1.735 \mathrm{de}$ & $0.415 f g$ \\
\hline $75 \% C+100 \% \mathrm{~K}+100 \% \mathrm{P}+$ Bio-F. & $2.113 \mathrm{bc}$ & $0.242 \mathrm{~cd}$ & $1.580 \mathrm{~b}$ & $1.712 \mathrm{fg}$ & $0.721 b$ \\
\hline $75 \% \mathrm{C}+75 \% \mathrm{~K}+75 \% \mathrm{P}+$ Bio-F. & $2.163 b$ & $0.235 \mathrm{~cd}$ & $1.597 \mathrm{~b}$ & $1.900 \mathrm{a}$ & $0.674 \mathrm{bc}$ \\
\hline $75 \% \mathrm{C}+50 \% \mathrm{~K}+50 \% \mathrm{P}+$ Bio-F. & $2.043 \mathrm{~cd}$ & $0.379 \mathrm{~b}$ & $1.631 \mathrm{ab}$ & $1.851 \mathrm{~b}$ & $0.494 \mathrm{ef}$ \\
\hline $75 \% C+25 \% \mathrm{~K}+25 \% \mathrm{P}+$ Bio-F. & $1.663 \mathrm{~g}$ & $0.202 d$ & $1.602 b$ & $1.821 \mathrm{c}$ & $0.675 \mathrm{bc}$ \\
\hline $50 \% \mathrm{C}+100 \% \mathrm{~K}+100 \% \mathrm{P}+\mathrm{Bio}-\mathrm{F}$. & $2.071 \mathrm{c}$ & $0.129 \mathrm{~d}$ & $1.671 \mathrm{a}$ & $1.829 \mathrm{c}$ & $0.572 d$ \\
\hline $50 \% \mathrm{C}+75 \% \mathrm{~K}+75 \% \mathrm{P}+\mathrm{Bio}-\mathrm{F}$. & $2.104 \mathrm{bc}$ & $0.166 \mathrm{~d}$ & $1.608 \mathrm{~b}$ & $1.825 \mathrm{c}$ & $0.656 \mathrm{c}$ \\
\hline $50 \% C+50 \% \mathrm{~K}+50 \% \mathrm{P}+$ Bio-F. & $1.905 \mathrm{e}$ & $0.139 d$ & $1.448 \mathrm{c}$ & $1.822 \mathrm{c}$ & $0.722 b$ \\
\hline \multirow[t]{2}{*}{$50 \% \mathrm{C}+25 \% \mathrm{~K}+25 \% \mathrm{P}+\mathrm{Bio}-\mathrm{F}}$. & $2.291 \mathrm{a}$ & $0.352 b$ & $1.591 \mathrm{~b}$ & $1.854 \mathrm{c}$ & $0.825 a$ \\
\hline & \multicolumn{5}{|c|}{ Third season: 2014} \\
\hline Control & $1.593 \mathrm{~h}$ & $0.132 \mathrm{~h}$ & $1.470 f$ & $1.821 \mathrm{c}$ & $0.400 \mathrm{e}$ \\
\hline $100 \% \mathrm{C}+100 \% \mathrm{~K}+100 \% \mathrm{P}+$ Bio-F. & $1.959 f$ & $0.211 \mathrm{e}$ & $1.591 \mathrm{e}$ & $1.829 \mathrm{bc}$ & $0.498 d$ \\
\hline $100 \% \mathrm{C}+75 \% \mathrm{~K}+75 \% \mathrm{P}+$ Bio-F. & $2.125 d$ & $0.162 \mathrm{fg}$ & $1.694 b$ & $1.835 \mathrm{bc}$ & $0.404 \mathrm{e}$ \\
\hline $100 \% \mathrm{C}+50 \% \mathrm{~K}+50 \% \mathrm{P}+$ Bio-F. & $2.386 a b$ & $0.212 \mathrm{e}$ & $1.774 a$ & $1.855 a$ & $0.499 d$ \\
\hline $100 \% \mathrm{C}+25 \% \mathrm{~K}+25 \% \mathrm{P}+$ Bio-F. & $1.719 \mathrm{~g}$ & $0.336 \mathrm{~b}$ & $1.660 \mathrm{c}$ & $1.829 \mathrm{bc}$ & $0.463 \mathrm{de}$ \\
\hline $75 \% \mathrm{C}+100 \% \mathrm{~K}+100 \% \mathrm{P}+$ Bio-F. & $1.977 \mathrm{ef}$ & $0.158 \mathrm{fg}$ & $1.631 \mathrm{~cd}$ & $1.822 \mathrm{c}$ & $1.053 a$ \\
\hline $75 \%$ C + $75 \%$ K + $75 \%$ P + Bio-F. & $2.334 \mathrm{bc}$ & $0.252 d$ & $1.621 \mathrm{~d}$ & $1.839 a-c$ & $0.665 \mathrm{bc}$ \\
\hline $75 \% \mathrm{C}+50 \% \mathrm{~K}+50 \% \mathrm{P}+\mathrm{Bio}-\mathrm{F}$. & $2.282 \mathrm{c}$ & $0.307 \mathrm{c}$ & $1.661 \mathrm{c}$ & $1.838 \mathrm{a}-\mathrm{c}$ & $0.514 d$ \\
\hline $75 \% \mathrm{C}+25 \% \mathrm{~K}+25 \% \mathrm{P}+$ Bio-F. & $1.752 \mathrm{~g}$ & $0.214 \mathrm{e}$ & $1.579 \mathrm{e}$ & $1.842 a b$ & $0.650 \mathrm{bc}$ \\
\hline $50 \% \mathrm{C}+100 \% \mathrm{~K}+100 \% \mathrm{P}+$ Bio-F. & $2.162 d$ & $0.164 f g$ & $1.642 \mathrm{~cd}$ & $1.791 \mathrm{~d}$ & $0.725 \mathrm{~b}$ \\
\hline $50 \% \mathrm{C}+75 \% \mathrm{~K}+75 \% \mathrm{P}+\mathrm{Bio}-\mathrm{F}$ & $1.752 \mathrm{~g}$ & $0.182 f$ & $1.480 f$ & $1.826 \mathrm{bc}$ & $0.625 c$ \\
\hline $50 \% \mathrm{C}+50 \% \mathrm{~K}+50 \% \mathrm{P}+\mathrm{Bio}-\mathrm{F}$. & $2.025 \mathrm{e}$ & $0.149 \mathrm{gh}$ & $1.561 \mathrm{e}$ & $1.835 \mathrm{bc}$ & $0.727 \mathrm{~b}$ \\
\hline $50 \% \mathrm{C}+25 \% \mathrm{~K}+25 \% \mathrm{P}+\mathrm{Bio}-\mathrm{F}$. & $2.402 a$ & $0.369 a$ & $1.580 \mathrm{e}$ & $1.855 a$ & $0.676 \mathrm{bc}$ \\
\hline
\end{tabular}

${ }^{*}$ C: Compost; K: Feldspar; P: Rock phosphate, and Bio-F.: Nitrobeine, Phosphorene, Potassein.

* Means within a column having the same letters are not significantly different according to Duncan`s Multiple Range Test at $5 \%$ level. 


\section{REFERENCES}

Adak, T.; Kumar, K.; Singha, A.; Shukla, S. K. and Singh, V. K. (2014). Assessing soil characteristics and guava orchard productivity as influenced by organic and inorganic substrates. J. of Animal and Plant Sci.; 24(4):1157-1165.

Akash Sharma Wali, V. K., Parshant Bakshi and Amit Jasrotia (2013). Effect of integrated nutrient management strategies on nutrient status, yield and quality of guava. Indian J. of Hort.; 70(3):333-339.

A.O.A.C. (1975). Association of Official Agricultural Chemists. "Official Methods of Analysis". $12^{\text {th }}$ Ed., published by AOAC, Washington D.C., USA.

A.O.A.C. (1995)..."Official Methods of Analysis". 16 ${ }^{\text {th }}$ Ed., Association of Official Analytical Chemists. International, Virginia, USA.

Baksh, H. ; Yadav, R. and Dwivedi, R. (2008). Effect of INM on growth, yield, yield attributing characters and quality of guava ( $P$. guajava $\mathrm{L}$.) cv. Sardar. Progressive Agric.; 8(2):141-144.

Devi, H. L.; Mitra, S. K. and Poi, S. C. (2014). Effect of different organic and biofertilizer sources on guava (Psidium guajava L.) 'Sardar'. Acta Hort.; (961):201-208.

Dewis, J. and Freitas, F. (1970). Physical and Chemical Methods of Soil and Water Analysis. Food and Agric. Org. of the U.N. (FAO), Soil Buletin No. 10.

Duncan, D. B. (1955). Multiple range and multiple F. tests. Biometrics, 11: 1-42.

Dutta, P. ; Maji, S. B. and Das, B. C. (2009). Studies on the response of biofertilizer on growth and productivity of guava. Indian J. of Hort.; 66(1):39-42.

El-Iraqy, M.A. (2014). Effect of biofertilizers and natural minerals on productivity and fruit quality of olive trees cv. "Picual". Inter. J. of Plant \& Soil Sci., 3 (11): 1387-1397.

El-Taweel, A. A. (2005). Studies on fertilization of guava trees. Ph.D.. Thesis Faculty of Agric. El.Mansoura Univ., Egypt.

Goswami, A. K.; Sant Lal and Misra, K. K. (2012). Integrated nutrient management improves growth and leaf nutrient status of guava cv. Pant Prabhat. Indian J. of Hort.; 69 (2):168-172.

Gowda, A. M.; El-Taweel, A. A. and Eassa, K. B. (2011). Studies on reducing the harmful effect of saline water irrigation on Picual olive trees. Minufiya J. Agric. Res., 36 (3): 623-645.

Horwitz, W. (1970). Official methods of Analysis. Association of Official Analytical Chemists, $11^{\text {th }}$ Ed., Washington D.C., USA.

Jackson, M.L. (1973). Soil Chemical Analysis. Prentice-Hall of India Private Limited M-97, New Delhi, India, 498pp.

Manning, D. A. (2010). Mineral sources of potassium for plant nutrition. A review article. Agronomy for sustainable develop., 30: 208-294.

Mead, R.; Curnow, R. N. and Harted, A. M. (1993). Statistical Methods in Agriculture and Experimental Biology. 2nd Ed., Chapman \& Hall Ltd., London, $335 \mathrm{pp}$. 
Meena, R. K., Mahwer, L. N. Sarolia, D. K. Saroj, P. L. Kaushik, R. A. (2013. Improving yield and nutrient status of rejuvenated guava orchard by integrated nutrient management under semi-arid conditions. Vegetos; 26 (1):233-242.

Mitra, S. K., Gurung, M. R. and Pathak, P. K. (2010). Integrated nutrient management in high density guava orchards. Acta Hort; (849):349-356.

Ram, R. A.; Bhriguvanshi, S. R.; Garg, N. and Pathak, R. K. (2007). Studies on organic production of guava (Psidium guajava L.) Cv. Allahabad Safeda. Acta Hort. (735):373-379.

Rubee Lata, D. H.; Ram, R. B. and Meena, M. L. (2011). Response of organic substrates on growth, yield and physiochemical characteristics of guava cv. Red fleshed. Indian J. of Ecology; 38 (1): 81-84.

SAS, Institute. (1994). SAS/STAT User's Guides Statistics. Vers. 6.04, $4^{\text {th }}$ Ed., SAS. Institute Inc. Cary, N.C., USA.

Shalini, A. K.; Mahawer, L. N. and Rajvir Sharma, H. L. (2010). Standardization of pruning intensity and integrated nutrient management in meadow orcharding of guava (Psidium guajava L.). Indian J. of Agric. Sci.; 80(8):673-678.

Subba, Rao, N. S. (1993). Biofertilizers in Agriculture. $3^{\text {rd }}$ Ed., Oxford \& IBH Publishing Co., Ltd., New Delhi, Bombay, Calcutta, 242pp.

Trivedi, Y. V.; Patel, N. L.; Ahlawat, T. R.; Gaikwad, S. S. and Bhalerao, P. P. (2012). Impact of organic manures and inorganic fertilizers on growth, yield, nutrient uptake and soil nutrient status in guava. Indian J. of Hort.; 69(4):501-506.

Wide, S. .; Corey, R. B.; Lyer, J. G. and Vioget, G. (1985). Soil and Plant Analysis for Tree Culture, $3^{\text {rd }}$ Ed., Oxford, IBH Publishing Co., New Delhi, pp. 93-116.

Willer, H. and Kilcher, L. (eds.) (2010). The World of Organic Agriculture. Statistics and Emerging Trends. FiBL, Frick and IFOAM Bonn.

Wilson, C. W. (1980). Guava, P. 279-299. in Nagy, S. and Shaw, P.E. (eds.) Tropical and sub-tropical fruits: Composition, properties and uses: Avl. Wastport, Conn. 
تأثير بعض معاملات التسميد على نمو وإنتاج وجودة ثمار الجوافة (صنف عتمانى).

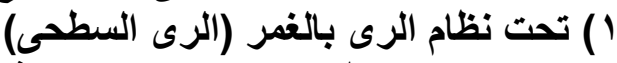

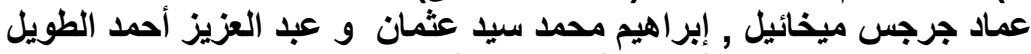

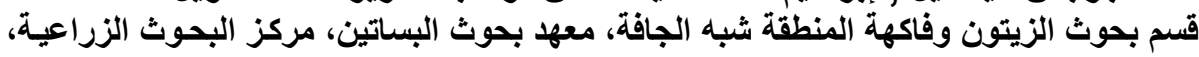
الجيزة، مصر.

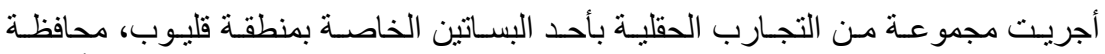

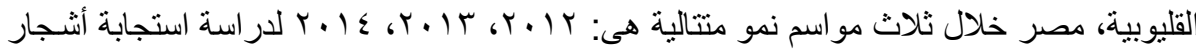

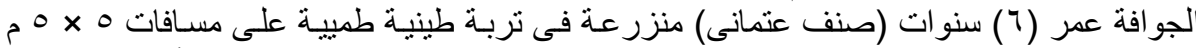

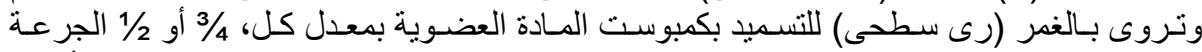

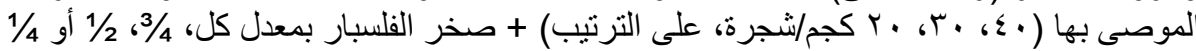

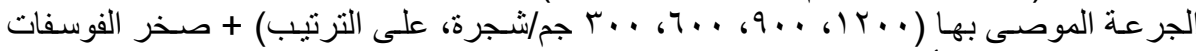

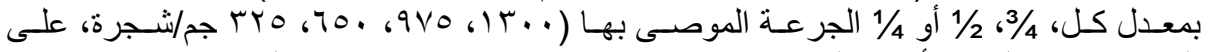

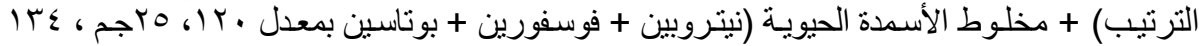

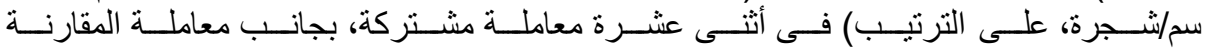
(بدون تسميد إضافى).

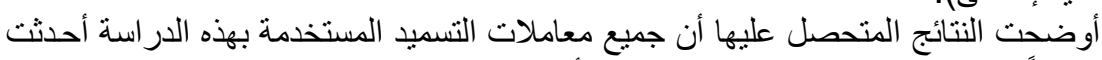

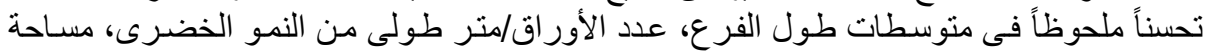

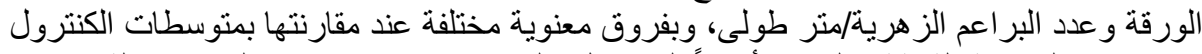

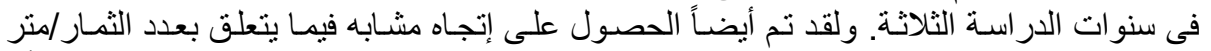

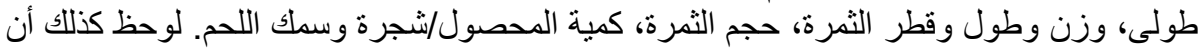

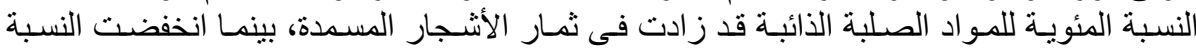

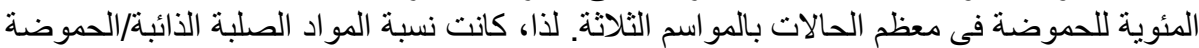

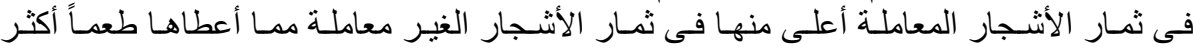

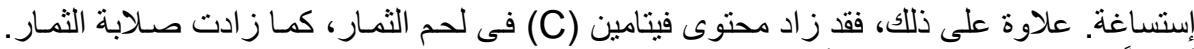

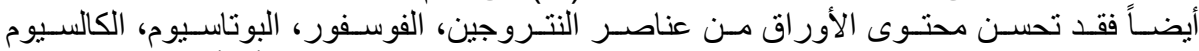

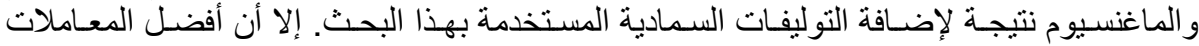

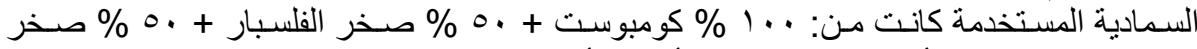

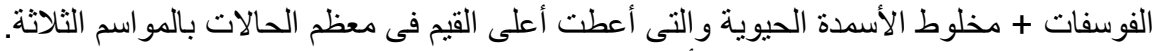

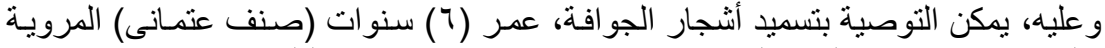

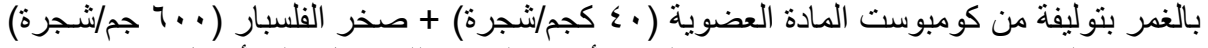

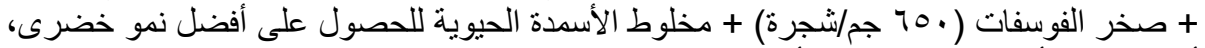
أعلى إنتاج و أفضل جودة للثنار بأقل تكلفة تجارية. 
Table (4): Effect of fertilization treatments on some vegetative growth traits and No. flower buds of (Psidium guajava L.) "Etmani" cv. tree under ordinary irrigation system during 2012, 2013 and 2014 seasons.

\begin{tabular}{|c|c|c|c|c|c|c|c|c|c|c|c|c|}
\hline \multirow{2}{*}{ Treatments } & \multicolumn{3}{|c|}{ Shoot length $(\mathrm{cm})$} & \multicolumn{3}{|c|}{ No. of leaves/lm } & \multicolumn{3}{|c|}{ Leaf area $\left(\mathrm{cm}^{2}\right)$} & \multicolumn{3}{|c|}{ No. flower buds/lm } \\
\hline & 2012 & 2013 & 2014 & 2012 & 2013 & 2014 & 2012 & 2013 & 2014 & 2012 & 2013 & 2014 \\
\hline Control & $15.30 \mathrm{e}$ & $17.23 \mathrm{bc}$ & $16.97 f$ & $60.32 \mathrm{~g}$ & $53.83 \mathrm{~g}$ & $55.40 f$ & $30.89 \mathrm{ef}$ & $33.73 f$ & $33.97 \mathrm{de}$ & $32.83 \mathrm{bc}$ & $28.25 \mathrm{e}$ & $29.66 \mathrm{~h}$ \\
\hline $100 \% \mathrm{C}+100 \% \mathrm{~K}+100 \% \mathrm{P}+$ Bio-F & 16.33ab & $18.13 a$ & $18.03 a b$ & $65.55 f$ & $62.69 f$ & $63.96 \mathrm{e}$ & $33.17 \mathrm{c}-\mathrm{e}$ & $35.39 \mathrm{ef}$ & $35.96 \mathrm{~d}$ & $32.29 \mathrm{~b}$ & $32.73 c$ & $33.64 \mathrm{fg}$ \\
\hline $100 \% \mathrm{C}+75 \% \mathrm{~K}+75 \% \mathrm{P}+$ Bio-F. & 15.73c-e & $17.23 \mathrm{bc}$ & $17.57 \mathrm{~b}-\mathrm{e}$ & $93.94 \mathrm{c}$ & $90.15 \mathrm{c}$ & $89.00 \mathrm{~b}$ & $33.94 \mathrm{~b}-\mathrm{d}$ & $47.03 \mathrm{~b}$ & $50.38 a b$ & $31.80 \mathrm{c}-\mathrm{e}$ & $35.58 \mathrm{a}$ & $35.85 \mathrm{~cd}$ \\
\hline $100 \% \mathrm{C}+50 \% \mathrm{~K}+50 \% \mathrm{P}+$ Bio-F. & $16.53 a$ & $18.00 \mathrm{a}$ & $17.50 \mathrm{c}-\mathrm{e}$ & $106.90 \mathrm{a}$ & $97.90 \mathrm{ab}$ & 68.14de & $48.07 a$ & $49.24 b$ & $51.49 a b$ & $36.05 a$ & $35.79 a$ & $37.90 \mathrm{a}$ \\
\hline $100 \% \mathrm{C}+25 \% \mathrm{~K}+25 \% \mathrm{P}+$ Bio-F. & $16.20 \mathrm{a}-\mathrm{c}$ & $17.40 \mathrm{bc}$ & 17.73a-e & $100.70 b$ & $97.43 a b$ & $96.19 a$ & $30.76 f$ & $39.68 \mathrm{c}$ & $40.16 \mathrm{c}$ & 31.41de & $31.23 d$ & $33.11 \mathrm{~g}$ \\
\hline $75 \% \mathrm{C}+100 \% \mathrm{~K}+100 \% \mathrm{P}+$ Bio-F. & $16.10 a-c$ & 17.67ab & $17.87 a-d$ & $93.60 \mathrm{c}$ & $73.78 d$ & $74.09 \mathrm{c}$ & $46.52 a$ & $48.64 b$ & $51.79 a b$ & $32.92 \mathrm{bc}$ & $34.87 \mathrm{ab}$ & $37.21 \mathrm{ab}$ \\
\hline $75 \% \mathrm{C}+75 \% \mathrm{~K}+75 \% \mathrm{P}+$ Bio-F. & $15.83 \mathrm{~b}-\mathrm{d}$ & $17.47 \mathrm{bc}$ & $17.93 a-d$ & $76.85 d$ & $69.10 \mathrm{e}$ & $70.19 \mathrm{~cd}$ & $35.19 \mathrm{bc}$ & $37.84 \mathrm{~cd}$ & $38.33 \mathrm{c}$ & $32.21 \mathrm{~b}-\mathrm{e}$ & $33.33 \mathrm{c}$ & $35.75 \mathrm{~cd}$ \\
\hline $75 \% C+50 \% \mathrm{~K}+50 \% \mathrm{P}+$ Bio-F. & 15.47de & $16.93 \mathrm{c}$ & 17.33 ef & $97.66 \mathrm{~b}$ & $99.07 a$ & $97.94 a$ & $48.66 \mathrm{a}$ & $51.53 a$ & $52.36 a$ & $32.33 b-d$ & $35.64 a$ & $37.92 a$ \\
\hline $75 \% \mathrm{C}+25 \% \mathrm{~K}+25 \% \mathrm{P}+$ Bio-F. & $16.40 \mathrm{a}$ & $17.30 \mathrm{bc}$ & $17.47 \mathrm{~d}-\mathrm{f}$ & $73.04 \mathrm{e}$ & $71.92 d$ & $72.80 \mathrm{c}$ & $36.17 \mathrm{~b}$ & $47.66 \mathrm{~b}$ & $32.12 \mathrm{e}$ & 31.03de & $32.73 \mathrm{c}$ & 35.27de \\
\hline $50 \% \mathrm{C}+100 \% \mathrm{~K}+100 \% \mathrm{P}+\mathrm{Bio}-\mathrm{F}$ & $16.53 a$ & $17.70 \mathrm{ab}$ & $18.17 a$ & $65.29 f$ & $64.59 f$ & $63.85 \mathrm{e}$ & $33.60 \mathrm{~cd}$ & $38.24 \mathrm{~cd}$ & $39.21 \mathrm{c}$ & $31.85 \mathrm{c}-\mathrm{e}$ & $33.72 \mathrm{c}$ & $33.77 \mathrm{fg}$ \\
\hline $50 \% \mathrm{C}+75 \% \mathrm{~K}+75 \% \mathrm{P}+$ Bio-F. & 16.37ab & $17.47 \mathrm{bc}$ & $18.00 \mathrm{a}-\mathrm{c}$ & $91.46 \mathrm{c}$ & $89.13 c$ & $87.24 b$ & $35.35 \mathrm{bc}$ & $36.85 \mathrm{de}$ & $38.31 \mathrm{c}$ & 30.96de & $33.40 \mathrm{c}$ & $34.64 \mathrm{ef}$ \\
\hline $50 \% \mathrm{C}+50 \% \mathrm{~K}+50 \% \mathrm{P}+$ Bio-F. & $16.10 \mathrm{a}-\mathrm{c}$ & $17.20 \mathrm{bc}$ & $17.60 \mathrm{~b}-\mathrm{e}$ & $98.37 \mathrm{~b}$ & $95.21 \mathrm{~b}$ & $93.98 \mathrm{a}$ & $48.14 a$ & $46.95 b$ & $50.97 a b$ & $32.09 \mathrm{~b}-\mathrm{e}$ & 33.95bc & $36.75 \mathrm{bc}$ \\
\hline $50 \% C+25 \% K+25 \% P+$ Bio-F. & 15.53de & $17.30 \mathrm{bc}$ & $17.47 \mathrm{~d}-\mathrm{f}$ & 76.24de & $96.93 \mathrm{ab}$ & $97.75 a$ & $32.06 \mathrm{~d}-\mathrm{f}$ & $30.89 \mathrm{~g}$ & $49.60 \mathrm{~b}$ & $30.86 \mathrm{e}$ & $33.40 \mathrm{c}$ & $35.09 \mathrm{de}$ \\
\hline
\end{tabular}

* C: Compost; K: Feldspar; P: Rock phosphate, Bio-F.: Nitrobeine, Phosphorene, Potassein and Im: Longitudinal meter.

${ }^{*}$ Means within a column having the same letters are not significantly different according to Duncan`s Multiple Range Test at $5 \%$ level. 